A - Research concept and design

B - Collection and/or assembly of data

$\mathrm{C}$ - Data analysis and interpretation

D - Writing the article

E - Critical revision of the article

F - Final approval of article

\section{Evaluation of the upper extremity functions of the non-affected side in hemiplegic patients during one month follow-up}

\author{
Nilsu Küçük ${ }^{1, B-D}$ (D), Ali Veysel Özden²,A-D (D), Kerem Alptekin*3,E-F (D) \\ ${ }^{1}$ Bahcesehir University, Turkey \\ ${ }^{2}$ Bahcesehir University, Vocational School of Health Sciences, Turkey \\ ${ }^{3}$ Bahcesehir University, Health Sciences Faculty, Turkey
}

${ }^{*}$ Correspondence: Kerem Alptekin; Bahcesehir University Health Sciences Faculty, Turkey; email: kalptekin79@hotmail.com

\begin{abstract}
Introduction: The aim of this study was to investigate the functional changes in the upper extremity of the intact side of hemiplegic patients within 0-12 months after stroke.

Materials and methods: The study included 32 individuals with Stage 3 and Stage 4 progression according to Brunnstrom stages, whose right side was dominant. The age range of the individuals in the study was $45-80$ years. This research was a prospective study planned according to the single group pretest-posttest pattern. There was no control group.

Results: As a result of the study, it was determined that pain increased in the shoulder region of the non-affected side during the one-month follow-up period and therefore, upper extremity functionality decreased. After one month, strength values of grip, shoulder flexion (left intact), shoulder extension, shoulder abduction (right intact), shoulder internal rotation, shoulder external rotation (left intact), elbow flexion, elbow extension, and wrist extension increased. Furthermore, during this follow-up period upper extremity functionality decreased, pain values increased in the shoulder area, and hand grip strength values increased in individuals whose disease duration was 0-6 months and 7-12 months.

Conclusion: The non-affected upper extremity of a stroke patient plays an important role in helping them throughout the activities of their daily life. This study concluded that, upper extremities of the non-affected side of hemiplegic patients are also affected in terms of muscle strength, pain and functionality.
\end{abstract}

Keywords: functionality, hemiplegia, pain, strength, upper extremity

\section{Introduction}

Cerebrovascular Accident (CVA) is a stroke that results from the sudden occlusion of blood vessels or bleeding in the brain and the resulting lesion causes certain effects in the relevant body part. Patients who survive CVA may develop hemiplegia (stroke) depending on the severity of the lesion. Hemiplegia is a condition in which part of the body is affected and is accompanied by neuromuscular dysfunctions and damage to muscular strength and sense [1-5]. Functional recovery after stroke is affected by many factors such as age of the patient, initial severity of the stroke, and lesion side [4].

Stroke involves the rapid loss of brain function due to a disturbance in blood flow to the brain and often affects upper extremity mobility [6]. In the majority of 
patients with stroke (more than 50\%), functional disability occurs in the upper extremity. As a result, patients may develop shoulder pain [7]. For these reasons, patients may not be able to use their affected upper extremity. Furthermore, workload will increase on the unaffected upper extremity. Even though there is motor control in the affected upper extremity after a stroke, patients tend to use the unaffected side more. Thus, they often perform daily activities with their unaffected upper limbs [8]. Under these circumstances, there may also be changes in the functional state of the intact upper extremity [9].

The literature contains many studies about shoulder pain, upper extremity functionalities, and the evaluation of muscle strength in hemiplegic patients [10,11]. Most of the studies evaluate upper extremity functionality of the affected side [10]. Additionally, parameters such as muscle strength, grip strength, pain level, joint range of motion, and functional capacity in the unaffected extremities of hemiplegic patients depend on non-use or less use of the affected side. In this regard, the number of studies investigating the functional changes in the intact upper extremity associated with the affected side is rather low [12-15].

The aim of this study is to investigate the functional changes in the upper extremity of the intact side of hemiplegic patients within 0-12 months after stroke occurred, to determine whether these changes are significant, and to establish a clinical bottom line based on the results. The outcome of the study may increase attention towards the unaffected side of hemiplegic patients and contribute to reorganization of their treatment and rehabilitation programs.

\section{Materials and methods}

This research was a prospective study planned according to the single group pretest-posttest pattern. There was no control group. The researchers selected participants from patients who were diagnosed with hemiplegia in a private rehabilitation clinic and were eligible to participate voluntarily in the study.

To determine the sample size, PS 3.5 packaged software was used for power analysis. This study was taken as a basis 80 percent power value and 0.01 alpha meaning level ratio according to the article titled' Grip Strength is a Representative Measure of Muscle Weakness in the Upper Extremity after Stroke'. As a result of the power analysis, it was decided to work with an experimental group of at least 7 people. More participants were included in the study: 32 volunteers aged between $45-80$ with a hemiplegic history of $0-12$ months.
The study was conducted only among individuals with a dominant right upper extremity because individuals with a dominant left upper extremity were not found.

\section{Criteria for Inclusion in the Study}

a) hemiplegic history of 0-12 months

b) no secondary disability or disease

d) good mental level and cooperative ability

e) aged between $45-80$ years

f) Stage 2 and above for Upper and Lower Extremity in Brunnstrom Stages

Age, height, body weight, affected side, dominant hand, duration of the disease and its stage according to the Brunnstrom Stages were recorded in the first examination of the patients. During the evaluation, grip strength was measured with a hand dynamometer and upper extremity muscle strength was measured with an isometric dynamometer. Range of Motion (ROM) was evaluated with a goniometer.

The GRIP-D dynamometer was used to measure the grip strength of the patients participating in the study. The patients were asked to hold the hand dynamometer with their unaffected hand and to sqeeze the device for 3 seconds with maximimal force. The grip strength measurement of the unaffected side was then read and recorded. Shoulder (shoulder flexion, extansion, abduction, adduction, internal rotation and external rotation), elbow (elbow flexion and extansion) and wrist (wrist flexion and extansion) joint range of motion values of the patients' unaffected upper extremities were measured using a universal goniometer. The upper extremity isometric muscle strength of the patients was measured with a microfet digital dynamometer. Shoulder flexion, extension, abduction, internal-external rotation, elbow flexion-extension, and wrist extension were measured.

The Visual Analogue Scale (VAS) was used in the assesment of pain. VAS was used to assess shoulder pain on the unaffected side of the participants. The patients were asked to mark the degree of pain they felt on a straight line numbered from 0 to 10 , with 0 being no pain, and 10 being the most severe pain [16] .

The Disabilities of the Arm, Shoulder, and Hand (DASH) Questionnaire was used to evaluate the upper extremity function of the patients. The DASH is a questionnaire that measures physical function in upper extremity disorders. The validity and reliability of the questionnaire in Turkish language was determined previously. Disability increases with high scores.

Patients participating in the study did not receive any rehabilitative treatment during the one-month follow-up period. They only continued their medication for stroke. The data collection process was completed by the second measurements one month after the initial 
measurements were taken. It was thought that 1-month follow-up would be enough to detect changes in the upper extremities of the unaffected side.

The Clinical Ethical Committee of Bahcesehir University approved on 20 December 2017 with file number 20/06. Informed consent of all patients was obtained as written.

\section{Statistical analysis}

The data obtained in this study was analyzed using statistical tests and assessments through IBM SPSS 22 packaged software and reported according to the scientific requirements of the statistical tests and analyses. Since the sample size included more than 30 patients
( $n>30)$, parametric methods were preferred for data analysis in this study. More specifically, the Related Samples T-Test was used in data analysis.

\section{Results}

The characteristic of the study participants is presented in table 1 .

As seen in table 2, a statistically significant diference was determined between upper extremity functionality values, pain values, and hand grip strength values as a result of the related sample t-test conducted in the research. After the one month follow-up period, upper

Tab. 1. Demographic findings

\begin{tabular}{lccc}
\hline & & Number & \% (percentage) \\
\hline \multirow{2}{*}{ Sex } & Male & 23 & 71.9 \\
& Famale & 9 & 28.1 \\
Age & $45-64$ & 15 & 46.9 \\
& $65-80$ & 17 & 53.1 \\
Dominant Upper Extremity & Right & 32 & 100.0 \\
& Left & 0 & 0.0 \\
Affected Upper Extremity & Right & 17 & 53.1 \\
& Left & 15 & 46.9 \\
Duration of Disease & $0-6$ Months & 13 & 40.6 \\
& $7-12$ Months & 19 & 59.4 \\
Stage of Disease & Stage 3 & 17 & 53.1 \\
& Stage 4 & 15 & 46.9 \\
Presence of a Systemic Disease & Yes & 20 & 62.5 \\
& No & 12 & 37.5 \\
History of Surgery in the Upper Extremity & Yes & 2 & 6.3 \\
& No & 30 & 93.8 \\
\hline Total & & 32 & 100 \\
\hline
\end{tabular}

Tab. 2. Analysis of the differences between pre-test and post-test results of functionality, pain and hand grip according to the intact upper extremity assessments

\begin{tabular}{|c|c|c|c|c|}
\hline & & Pre-test & Post-test & $\mathrm{p}$ \\
\hline & & Avg. \pm s.d & 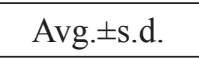 & \\
\hline \multirow{2}{*}{$\begin{array}{l}\text { Upper Extremity Functionality } \\
\text { (DASH) }\end{array}$} & Left & $18.23 \pm 13.05$ & $27.67 \pm 18.73$ & .007 \\
\hline & Right & $13.19 \pm 12.22$ & $16.11 \pm 14.13$ & .039 \\
\hline \multirow{2}{*}{ Pain in Shoulder (VAS) } & Left & $1.29 \pm 1.65$ & $4.12 \pm 1.93$ & .000 \\
\hline & Right & $1.20 \pm 1.15$ & $2.80 \pm 2.37$ & .003 \\
\hline \multirow{2}{*}{ Hand Grip Strength } & Left & $24.88 \pm 10.95$ & $27.95 \pm 10.98$ & .001 \\
\hline & Right & $23.17 \pm 6.80$ & $25.47 \pm 6.32$ & .000 \\
\hline
\end{tabular}


extremity functionality decreased, pain values in the shoulder region increased, and hand grip strength values increased for the intact sides of the individuals.

As seen in table 3, as a result of the related sample $\mathrm{t}$ test conducted in the research, there was a statisticaly significant difference among shoulder flexion strength $(\mathrm{p}<.01)$ and shoulder external rotation strength $(\mathrm{p}<.01)$ values which were measured before and after the one-month follow-up period for the left side intact individuals. However, there was no statistically significant difference among shoulder flexion strength $(\mathrm{p}>.05)$ and shoulder external rotation strength $(p>.05)$ for the right side intact individuals.

There was no statistically significant difference between the shoulder abduction strength $(p>.05)$ values for the left side intact individuals, but there was a statistically significant difference for the right side intact individuals $(\mathrm{p}<.05)$. Since the right hand of all participants before the stroke was dominant, it was expected to see muscle strength increases, especially in the left-side intact patients, because they would start using their left hand more for activities of daily living. The increase in muscle strength on the left intact side was greater, but not statistically significant according to the right side increase. Mostly, it can be said that, regardless of the right or left side, muscle strength measurements increase statistically in the non-affected upper extremities.

As seen in Table 4, as a result of related sample T-test conducted in the research, there was a statisticaly significant difference among the shoulder flexion values which were measured before and after one month follow-up period for the right side intact individuals $(\mathrm{p}<.05)$. However, there was no statistically significant difference for the left side intact individuals $(\mathrm{p}>.05)$. There was no statistically significant difference among the shoulder internal rotation $(\mathrm{p}>.05)$, external rotation $(p>.05)$ and wrist flexion $(p>.05)$ values for the right side intact individuals. Contrarily, there was a statistically significant difference among the shoulder internal rotation $(p<.01)$, external rotation $(p<.05)$, and wrist flexion $(p<.05)$ values for the left side intact individuals. Joint range of motion tended to decrease in some directions on the non-affected side of the hemiplegic patients. Differences between the right and left intact sides may have occurred with the duration and severity of the disease.

As seen in table 5, a statistically significant diference was determined among upper extremity functionality values, pain values, and hand grip strength values measured before and after the one month follow-up period, according to therelated sample T-test conducted in

Tab. 3. Analysis of the differences between pre-test and post-test results of upper extremity strength according to the intact upper extremity assessments

\begin{tabular}{|c|c|c|c|c|}
\hline & & Pre-test & Post-test & $\mathrm{p}$ \\
\hline & & 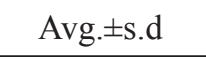 & Avg.土s.d. & \\
\hline \multirow{2}{*}{ Shoulder Flexion Strength } & Right & $46.74 \pm 34.11$ & $48.87 \pm 35.79$ & .154 \\
\hline & Left & $43.46 \pm 27.89$ & $49.50 \pm 33.21$ & .002 \\
\hline \multirow{2}{*}{ Shoulder Extension Strength } & Right & $46.78 \pm 33.78$ & $49.46 \pm 34.48$ & .001 \\
\hline & Left & $42.00 \pm 26.47$ & $48.72 \pm 29.36$ & .002 \\
\hline \multirow{2}{*}{ Shoulder Abduction Strength } & Right & $40.84 \pm 31.36$ & $44.24 \pm 32.94$ & .017 \\
\hline & Left & $41.01 \pm 26.51$ & $43.44 \pm 25.64$ & .098 \\
\hline \multirow{2}{*}{$\begin{array}{l}\text { Shoulder Internal Rotation } \\
\text { Strength }\end{array}$} & Right & $38.19 \pm 27.03$ & $42.82 \pm 31.54$ & .011 \\
\hline & Left & $38.75 \pm 31.02$ & $42.42 \pm 30.49$ & .002 \\
\hline \multirow{2}{*}{$\begin{array}{l}\text { Shoulder External Rotation } \\
\text { Strength }\end{array}$} & Right & $35.60 \pm 26.74$ & $35.80 \pm 23.74$ & .919 \\
\hline & Left & $35.31 \pm 22.83$ & $38.99 \pm 25.60$ & .004 \\
\hline \multirow{2}{*}{ Elbow Flexion Strength } & Right & $40.39 \pm 29.04$ & $47.45 \pm 33.80$ & .001 \\
\hline & Left & $46.14 \pm 33.23$ & $55.01 \pm 37.84$ & .002 \\
\hline \multirow{2}{*}{ Elbow Extension Strength } & Right & $37.27 \pm 28.18$ & $41.16 \pm 29.06$ & .033 \\
\hline & Left & $41.63 \pm 29.39$ & $44.75 \pm 28.81$ & .001 \\
\hline \multirow{2}{*}{ Wrist Extension Strength } & Right & $31.98 \pm 23.23$ & $39.53 \pm 24.89$ & .001 \\
\hline & Left & $30.97 \pm 20.01$ & $39.43 \pm 20.83$ & .001 \\
\hline
\end{tabular}


Tab. 4. Analysis of the differences between pre-test and post-test results of joint range of motion according to the intact upper extremity assessments

\begin{tabular}{|c|c|c|c|c|}
\hline & & Pre-test & Post-test & $\mathrm{p}$ \\
\hline & & Avg. \pm s.d & 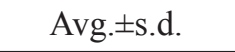 & \\
\hline \multirow{2}{*}{ Shoulder Flexion } & Left & $174.12 \pm 5.99$ & $170.71 \pm 5.54$ & .062 \\
\hline & Right & $174.93 \pm 5.73$ & $171.27 \pm 7.55$ & .016 \\
\hline \multirow{2}{*}{ Shoulder Extension } & Left & $56.06 \pm 4.64$ & $55.41 \pm 4.56$ & .428 \\
\hline & Right & $53.93 \pm 3.22$ & $54.40 \pm 4.03$ & .594 \\
\hline \multirow{2}{*}{ Shoulder Abduction } & Left & $172.65 \pm 7.53$ & $201.06 \pm 131.80$ & .392 \\
\hline & Right & $173.40 \pm 6.17$ & $170.87 \pm 8.56$ & .071 \\
\hline \multirow{2}{*}{ Shoulder Adduction } & Left & $43.76 \pm 2.66$ & $44.47 \pm 2.24$ & .062 \\
\hline & Right & $43.33 \pm 1.99$ & $43.93 \pm 2.05$ & .057 \\
\hline \multirow{2}{*}{ Shoulder Internal Rotation } & Left & $78.94 \pm 10.76$ & $74.18 \pm 9.36$ & .006 \\
\hline & Right & $77.67 \pm 7.46$ & $75.60 \pm 8.86$ & .109 \\
\hline \multirow{2}{*}{ Shoulder External Rotation } & Left & $79.18 \pm 9.58$ & $73.94 \pm 8.93$ & .017 \\
\hline & Right & $81.40 \pm 7.71$ & $78.87 \pm 9.36$ & .165 \\
\hline \multirow{2}{*}{ Elbow Flexion } & Left & $145.88 \pm 4.04$ & $146.29 \pm 3.29$ & .402 \\
\hline & Right & $147.00 \pm 3.91$ & $147.13 \pm 3.04$ & .846 \\
\hline \multirow{2}{*}{ Wrist Flexion } & Left & $73.18 \pm 5.58$ & $72.29 \pm 5.95$ & .020 \\
\hline & Right & $72.87 \pm 6.79$ & $73.47 \pm 5.94$ & .132 \\
\hline \multirow{2}{*}{ Wrist Extension } & Left & $66.41 \pm 3.02$ & $66.88 \pm 3.06$ & .203 \\
\hline & Right & $66.67 \pm 3.58$ & $67.00 \pm 3.42$ & .403 \\
\hline
\end{tabular}

Tab. 5. Analysis of the differences between pre-test and post-test results of functionality, pain and hand grip according to the duration of disease assessments

\begin{tabular}{lcccc}
\hline & Duration of disease & Pre-test & Post-test & p \\
\hline Upper Extremity Functionality & Months & Avg. \pm s.d & Avg. \pm s.d. & \\
(DASH) & $0-6$ & $14.13 \pm 15.20$ & $24.88 \pm 22.14$ & .013 \\
\multirow{2}{*}{ Pain in Shoulder (VAS) } & $7-12$ & $17.05 \pm 11.00$ & $20.45 \pm 13.83$ & .026 \\
& $0-6$ & $.69 \pm 1.44$ & $4.23 \pm 2.35$ & .000 \\
Hand Grip Strength & $7-12$ & $1.63 \pm 1.30$ & $3.00 \pm 2.03$ & .002 \\
& $0-6$ & $24.86 \pm 6.41$ & $28.04 \pm 7.03$ & .002 \\
& $7-12$ & $23.55 \pm 10.76$ & $25.94 \pm 10.31$ & .000 \\
\hline
\end{tabular}

the research, for the individuals with disease duration of $0-6$ months and individuals with disease duration 7-12 months.

Accordingly, upper extremity functionality decreased, pain values in the shoulder region increased, and hand grip strength values increased, for the individuals whose disease duration was $0-6$ months and 7-12 months.

\section{Discussion}

This study concluded that, the upper extremities of the intact side of hemiplegic patients with one year of disease duration are also affected in terms of muscle strength, pain, and functionality. We determined that, after the one-month follow-up period, pain level increased in the shoulder region of the intact arm, functionality 
decreased, and hand grip strength increased in all hemiplegic patients. Shoulder flexion, shoulder internal rotation, shoulder external motion, and wrist flexion range of motion decreased. Contrarily, muscle strength measurements generally increased after the one-month follow-up period. When the effect of disease duration (0-6 months or 7-12 months) on the parameters was evaluated, no statistically significant difference was found between the two groups.

The number of studies in the literature investigating the intact upper extremity in patients with stroke is quite low. Unlike the present study, Slijper et al. analyzed the effects of computer game based upper extremity exercises on patients diagnosed with stroke with 11 patients aged between 26-66, who had a stroke with duration of more than 6 months. They found that there was significant improvement in hand grip strength in the affected side after 16-18 weeks of exercise. No change, however, was observed in the unaffected side [17]. In our study, however, hand grip strength in the intact side increased during the course of the one-month follow-up period. It should be noted that the pre-test was completed at least 6 months after the stroke in the study of Slijper et al. However, participation in the current study occurred within 0-12 months. This may be because the increase in grip strength observed in the intact upper limb associated with stroke stops after a certain period.

In another study on central pain assessment in patients with stroke, Kılıç found a negative relationship between pain and functional status, while independent mobility of the patients decreased due to the increase in pain level [18]. Zimmerli et al. conducted a study with 10 participants in order to analyze the level of challenge in balance exercises on the affected and unaffected upper extremity after stroke. They determined that there was a significant difference between the performance levels of the patients during intermediate and more challenging exercises performed with the unaffected arm, while there was no significant difference in the arm affected by stroke [19]. Data in the literature demonstrate that there is overload on the unaffected side due to an inability to use the affected side, which results in an increase in pain level and limits individuals' functionality. In line with this information, it was observed in our study that the pain level increased according to VAS results in the upper extremity of the intact arm after the one-month follow-up period. Therefore, upper extremity functionality decreased. However, for the same reason, as a result of overload, muscle strength generally increased on the intact side.

In a study conducted by Ekstrand et al. with 44 patients who had a stroke at least 6 months previously, it was found that there was a directly proportional relationship among hand grip strength and shoulder abduction. Elbow flexion and extension strength in the upper extremity was affected less by stroke. This finding explains the parallelism in the relevant changes. In our study, it was also determined that hand grip strength and upper extremity strength on the unaffected side increased after one month of follow-up [20].

$\mathrm{Yi}$ et al. investigated the relationship between grip strength on the unaffected side and post-stroke functional recovery. Hand grip strength on both sides was measured twice for 127 patients after the stroke as well as prior to discharge from the hospital. The results showed that grip strength on the intact side had an impact on the short-term functional gain and recovery after stroke. The authors of the study stated that the patients with stronger grip strength on the intact side experienced faster recovery, and that the addition of the intact side to post-stroke rehabilitation exercises was effective in the recovery process. This proves the post-stroke increase in the grip strength on the intact side shown in the present study, in which we observed that grip strength increased in both right and left hemiplegic patients in the one-month followup period. The increase in hand grip strength on the intact side of right hemiplegia cases was found to be higher than left side hemiplegic patients. We believe that this result depends on the patient's dominant side. The dominant side of the 32 patients who participated in the study was previously the right side. In patients whose dominant side was affected by stroke, an increase was observed in the strength of the non-dominant (intact) extremity due to heavier use than the prestroke period [4].

In a study conducted by Balc1 et al., functionality, pain, and tactual sense between the intact upper extremities of patients with stroke and the intact upper extremities of healthy individuals were compared. Twenty healthy and 20 hemiplegic patients were included in the study. It was determined that the pain values were higher in the intact side upper extremities of the hemiplegic patients. This supports our observation of an increase in pain levels in the intact side upper extremities of the patients with stroke after the one-month follow-up period [6].

\section{Conclusion}

Our study showed that, pain level, function and hand grip strength has changed on the intact side of hemiplegic patients following a one-month follow-up period. We hope that the outcome of this study may increase attention towards the unaffected side of hemiplegic patients and contribute to reorganization of the treatment and rehabilitation programs of them in the future. 


\section{Funding}

This research received no external funding.

\section{Conflicts of interest}

The authors declare no conflict of interest.

\section{References}

1. Kilinc CS. The effect of body mass index on quality of life and activities of daily living in adult hemiplegic patients. Master Thesis. Izmir: Dokuz Eylul University Health Sciences Institute, 2014.

2. Adiguzel H. Evaluation of the effect of upper extremity functionality on walking in hemiplegic patients with shoulder pain and upper extremity spasticity. Master Thesis. Izmir: Dokuz Eylul University Health Sciences Institute, 2013.

3. Langhorne P, Duncan P. Does the organization of postacute stroke care really matter? Stroke. 2001; 32(1): 268-74.

4. Yi Y, Shim JS, Oh BM, Seo HG. Grip strength on the unaffected side as an independent predictor of functional improvement after stroke. Am J Phys Med Rehab. 2017; 96(9): 616-20.

5. Sahan M, Sebe A, Acikalin A, Akpinar O, Koc F, Ay $\mathrm{MO}$, et al. Acute-phase reactants and cytokines in ischemic stroke: do they have any relationship with short-term mortality? Eur Rev Med Pharmacol Sci. 2013; 17(20): 2773-7.

6. Balcı CN, Dogru E, Aytar A, Gokmen O, Depreli O. Comparison of upper extremity function, pain, and tactile sense between the uneffected side of hemiparetic patients and healthy subjects. J Phys Ther Sci. 2016; 7(28): 1998-2001.

7. Ozturk S, Akyol Y, Ulus Y, Tander B, Kuru O. Determinants of disease specific health-related quality of life in stroke patients. J PMR Sci. 2018; 21(3): 107-14.

8. Koyuncu E, Nakipoglu GF, Dogan A, Ozgirgin N. The effectiveness of functional electrical stimulation for the treatment of shoulder subluxation and shoulder pain in hemiplegic patients: A randomized controlled trial. Disabil Rehabil. 2010; 32(7): 560-6.

9. Zhang L, Han X, Li P, Liu Y, Zhu Y, Zou J, Yu Z. A study of tapping by the unaffected finger of patients presenting with central and peripheral nerve damage. Front Hum Neurosci. 2015; 9(260). doi: 10.3389/ fnhum.2015.00260.
10. Paci M, Nannetti L, Rinaldi LA. Glenohumeral subluxation in hemiplegia: An overview. J Rehabil Res Dev. 2005; 42(4): 557-68.

11. Price CL, Rodgers H, Franklin P. Glenohumeral subluxation, scapula resting position and scapula rotation after stroke; non-invasive evaluation. Arch Phys Med Rehabil. 2001; 82(7): 955-60.

12. Bayram A. The relationship between shoulder pain and upper extremity functions in hemiplegia. Medical Speciality Thesis. Konya: Selçuk University Faculty of Medicine, Department of Physical Medicine and Rehabilitation.1997.

13. Yoo C, Suh S, Kim Y. Analysis of affected and nonaffected sides of stroke hemiparalysis patients and correlations between rehabilitation therapy assessments using the bioelectrical impedance analysis method. J Phys Ther Sci. 2016; 28(12): 3306-9.

14. Ehrensberger M, Simpson D, Broderick P, Monaghan K. Cross-education of strength has a positive impact on post-stroke rehabilitation: A systematic literature review. Top Stroke Rehabil. 2016; 23(2): 126-35.

15. Osu R, Otaka Y, Ushiba J, Sakata S, Yamaguchi T, Fujiwara T, Kondo K, Liu M. A pilot study of contralateral homonymous muscle activity simulated electrical stimulation in chronic hemiplegia. Brain Inj. 2012; 26(9): 1105-12.

16. Carlsson AM. Assessment of chronic pain. I. Aspects of the Reliability and Validity of the Visual Analogue Scale. Pain. 1983; 16(1): 87-101.

17. Slijper A, Svensson KE, Backlund P, Engstrom H, Sunnerhagen KS. Computer game-based upper extremity training in the home environment in stroke persons: A single subject design. J Neuroeng Rehabil. 2014; 11(35). doi: 10.1186/1743-0003-11-35.

18. Kılıç Z, Erhan B, Gündüz B, Iska Elvan G. Central post-stroke pain in stroke patients: Incidence and the effect on quality of life. Turk J Phys Med Rehab. 2015; 61: 142-7.

19. Zimmerli L, Krewer C, Gassert R, Müller F, Riener R, Lunenburger L. Validation of a mechanism to balance exercise difficulty in robot-assisted upper-extremity rehabilitation after stroke. J Neuroeng Rehabil. 2012; 9(6). https://doi.org/10.1186/1743-0003-9-6.

20. Ekstrand E, Lexell J, Brogardh C. Grip strength is a representative measure of muscle weakness in the upper extremity after stroke. Top Stroke Rehabil. 2016; 23(6): 400-5. 